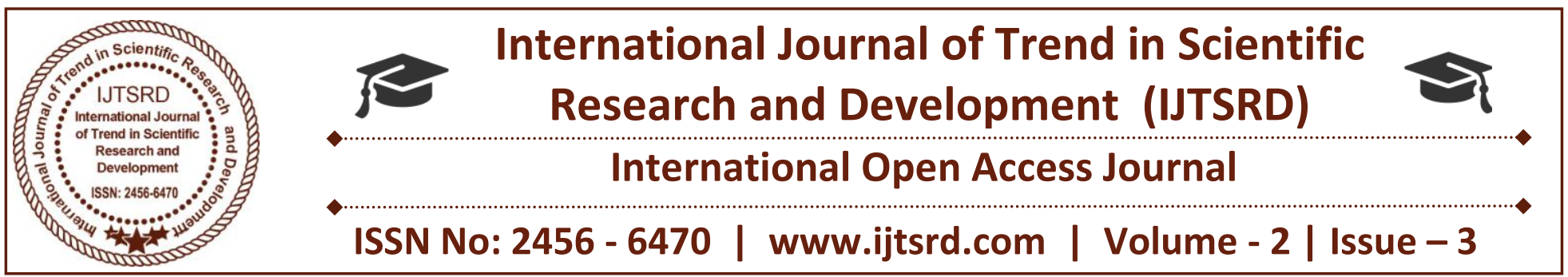

\title{
Challenges and Emerging Technology in 5G Network
}

\author{
Kiran Likhar, Shraddha Karale, Rashmi Bhat \\ Department of CSE, IT Institute of Rajiv Gandhi College \\ of Engineering \& Research (RGCER), TGPCET, Nagpur, India
}

\begin{abstract}
5G Mobile Wireless network system yield new challenges. In future the demand that need to be addressed are capacity, increase data rate and reduce latency. This paper focus on latest technologies used in wireless mobile communication network. In this, some emerging technologies use like device-to-device Communication (D2D), Interference Management, Spectrum sharing with cognitive radio, network cloud and Internet of Things (IoT), Massive MIMO. The IoT allows objects to be sensed or controlled remotely across existing network infrastructure and network cloud describing the access of networking resources from a centralized third-party provider.
\end{abstract}

Keywords: Wireless network, D2D, Network Cloud, Cognitive Radio, IoT

\section{INTRODUCTION}

\section{What is 5G?}

5 th generation mobile networks or 5th generation wireless systems, abbreviated $5 \mathrm{G}$, are the proposed next telecommunications standards beyond the current 4G/IMT-Advanced standards.5G will brings lot of new services to the use like amazing volume amazingly fast, always best connected, no perceived delay, massive amount of connected things and people, energy efficiency, flexible programmable network, Secure Network.5G Network look like Entire world with seamless communication between anybody ( people to people), Anything (people to machine, machine to machine), Anywhere (wherever they are), Anytime ( Whenever they need), Any How (Whatever Electronics).

\section{Need of 5G}

With increasing demand of user $4 \mathrm{G}$ is now replaced with $5 \mathrm{G}$.Mobile broadband access is expected to have a drastic increase with 1000 times(with data rates of up to $20 \mathrm{Gbps}$ ) more aggregate throughput and 10 times more at the link level from 2010 to 2020.Second, an increasing number of objects are being digitalized to form the Internet of Things, posing more stringent requirements on latency, battery lifetime, etc. To enhance service provisioning and satisfy the coming diversified requirements, it is necessary to revolutionize the cellular networks with cutting-edge technologies

\section{Challenges}

Fig 1 shows the evolution of $5 \mathrm{G}$ with challenges which include network requirements, wireless technology and spectrum.

\section{A. Network Requirement}

$5 \mathrm{G}$ networks will have to deliver baseline data speeds of $100 \mathrm{Mbit} / \mathrm{s}$ and peak speeds of up to $10 \mathrm{Gbit} / \mathrm{s}$. With wireless technologies already approaching the Shannon limit for bits/ $\mathrm{Hz}$ on individual radio links, the focus must turn to packing in more base stations in a given area, to achieve substantial increases in bits/Hz/km2 Dramatic increase in the number of devices to be supported will lead to vast numbers of machine-to-machine and person-to-machin machineto-machine and person-to-machine interactions, as part of the Internet of Things (IoT). This will require new approaches, to achieve reliable, efficient and 
secure communication without compromising the efficiency of other aspects of $5 \mathrm{G}$ networks.

\section{TABLE I: 5G Requirements}

\begin{tabular}{|c|c|}
\hline \multicolumn{2}{|c|}{ 5G requirements } \\
\hline \multirow[t]{4}{*}{$\begin{array}{l}\text { User } \\
\text { Perspective }\end{array}$} & $\begin{array}{l}\text { Ultra High Speed1000x(Anywhere } \\
\text { 1Gbps/user) }\end{array}$ \\
\hline & Ms latency \\
\hline & Realistic Content \\
\hline & Low Battery Consumption \\
\hline \multirow{4}{*}{$\begin{array}{l}\text { Performance } \\
\text { Perspective }\end{array}$} & Ultra High Capacity \\
\hline & $\begin{array}{l}\text { Massive Connectivity of } \\
\text { Devices }\end{array}$ \\
\hline & Traffic(IoT) \\
\hline & Secure Seamless Connectivity \\
\hline \multirow{3}{*}{$\begin{array}{l}\text { Architecture } \\
\text { Perspective }\end{array}$} & SW based Flexibility \\
\hline & Real time data analysis \\
\hline & Network as a service \\
\hline \multirow{2}{*}{$\begin{array}{l}\text { Operation } \\
\text { Perspective }\end{array}$} & High Reliability and security (>99\%) \\
\hline & Healing/Configuration \\
\hline
\end{tabular}

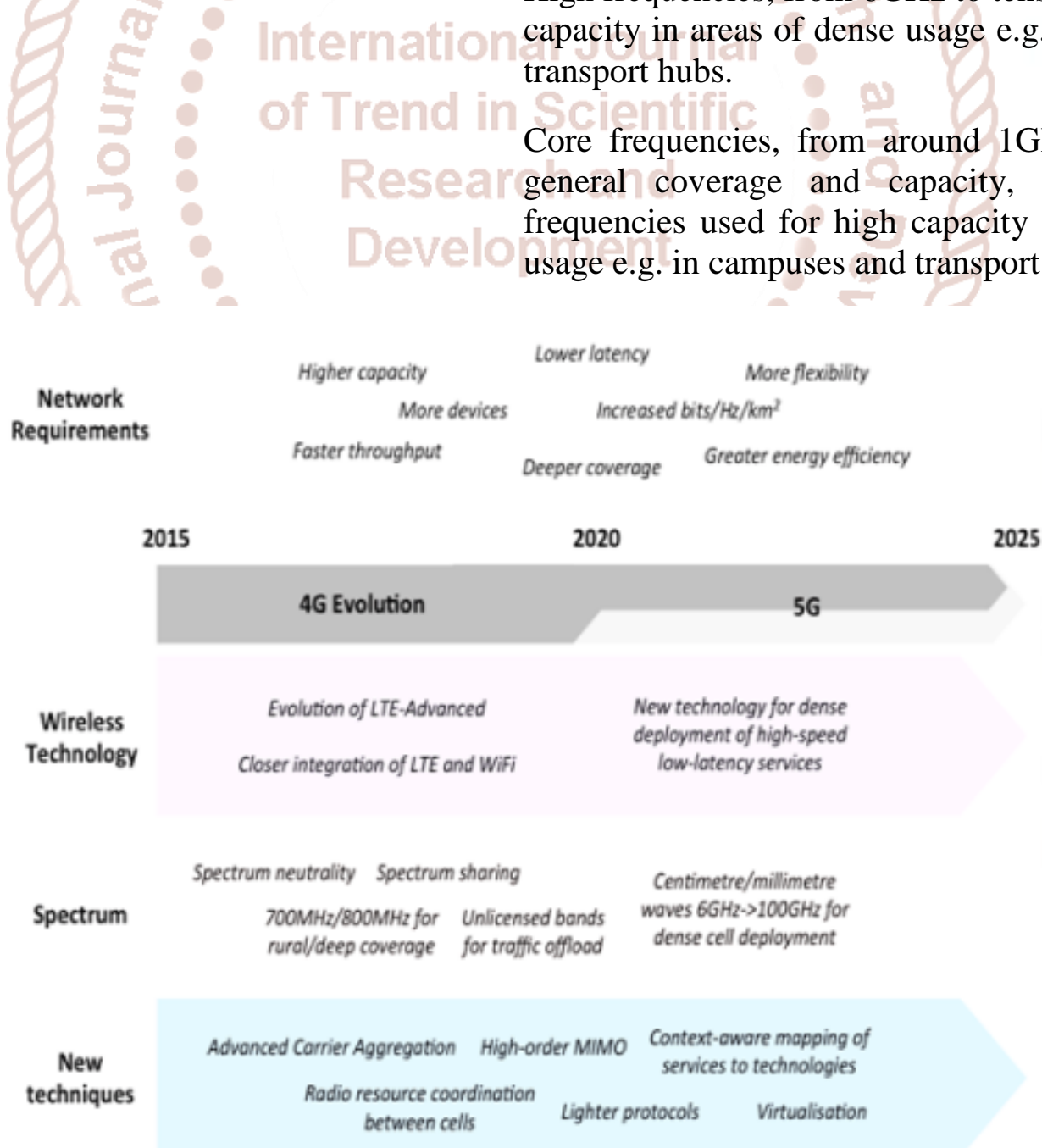

Fig. 1 Evolution of 5G 


\section{New techniques}

Certain approaches are already appearing in the context of $4 \mathrm{G}$ systems and are likely to evolve into $5 \mathrm{G}$ also Advanced Carrier Aggregation LTE will include the facility to combine disparate LTE carriers, which may or may not be in the same frequency band, to increase the peak user data rate and capacity of a network. The aim is to support up to five LTE carriers, each up to $20 \mathrm{MHz}$ bandwidth. Radio resource coordination between cells Coordinated Multipoint (CoMP) feature of LTE-Advanced, which turns the OFDMA scheme to advantage by deliberately sending and receiving data between a mobile device and multiple LTE-Advanced base stations. Network architectures will interplay between cells of different sizes, between licensed and unlicensed spectrum, between Frequency Division Duplex (FDD) and Time Division Duplex (TDD) connections, and between LTE-Advanced and WiFi .

\section{Key Technology}

It includes mainly Massive MIMO, Spatial Modulation, Cognitive Radio Network, Mobile Femto Cell,IoT

\section{A. Massive MIMO}

Massive MIMO can consist of number of Base Station (BSs), with the limited availability of spectrum and the projected massive number of IoT devices to come online, 5G networks will have to make far more efficient use of spectrum than that of wireless networks. Massive MIMO which is a form of multiuser MIMO in which the number of antennas at the base station is much larger than the number of mobile stations per signaling resource. The large number of base station antennas relative to the number of mobile stations results in a channel response that is quasiorthogonal and has the potential to yield huge gains in spectral efficiency. Such conditions would enable many more devices to be served with the same frequency and time resources within a given cell compared to modern-day 4G system. Massive MIMO eavesdropper fails to decode the original symbols while the legitimate users are able to recover original symbol with only limited number of antennas.

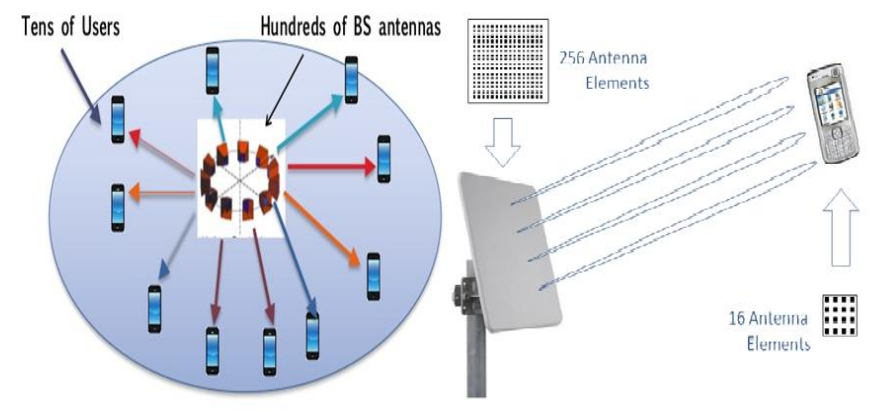

Fig. 2 Massive MIMO

Multiple transmitting and Receiving antennas Performance improvement is obtained in terms of reliability, spectral efficiency and energy efficiency.

\section{Issues}

Co-located or Distributed: Effect of noise and fast fading vanishes

Intracellular interference mitigated : using simple linear precoding and decoding Method

\section{B. Spatial Modulation}

SM encodes part of data into spatial position of each antenna in an array. Increases data rate compared to a single antenna system. Information Bits splits into two blocks of $\log 2(\mathrm{NB})$ and $\log 2(\mathrm{M})$. NB number of complex antennas and $\mathrm{M}$ size of complex constellation. First block identifies active antenna Second block selects symbol from the constellation. SM combination of Space shift keying and amplitude/Phase Modulation
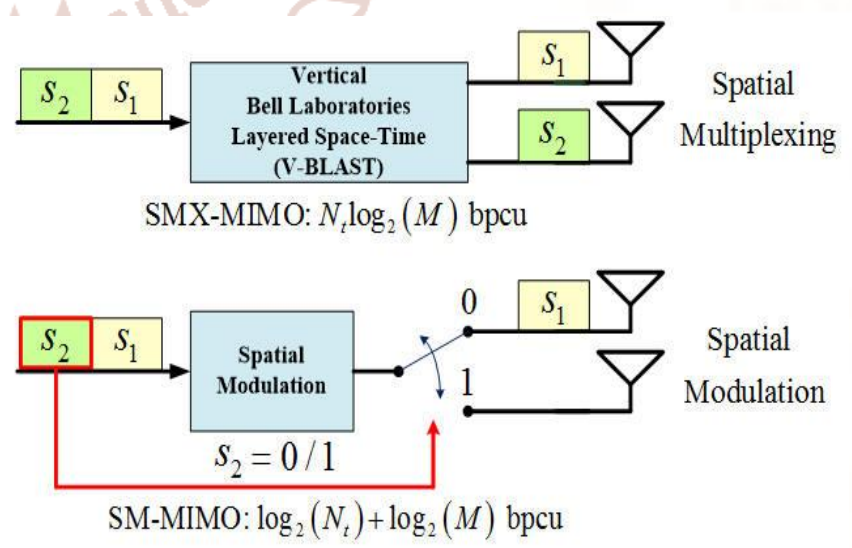

Fig 3: Spatial Modulation

SM can Mitigate three major issues in MIMO

Inter channel Interference

Inter antenna Synchronization

Multiple RF chains 


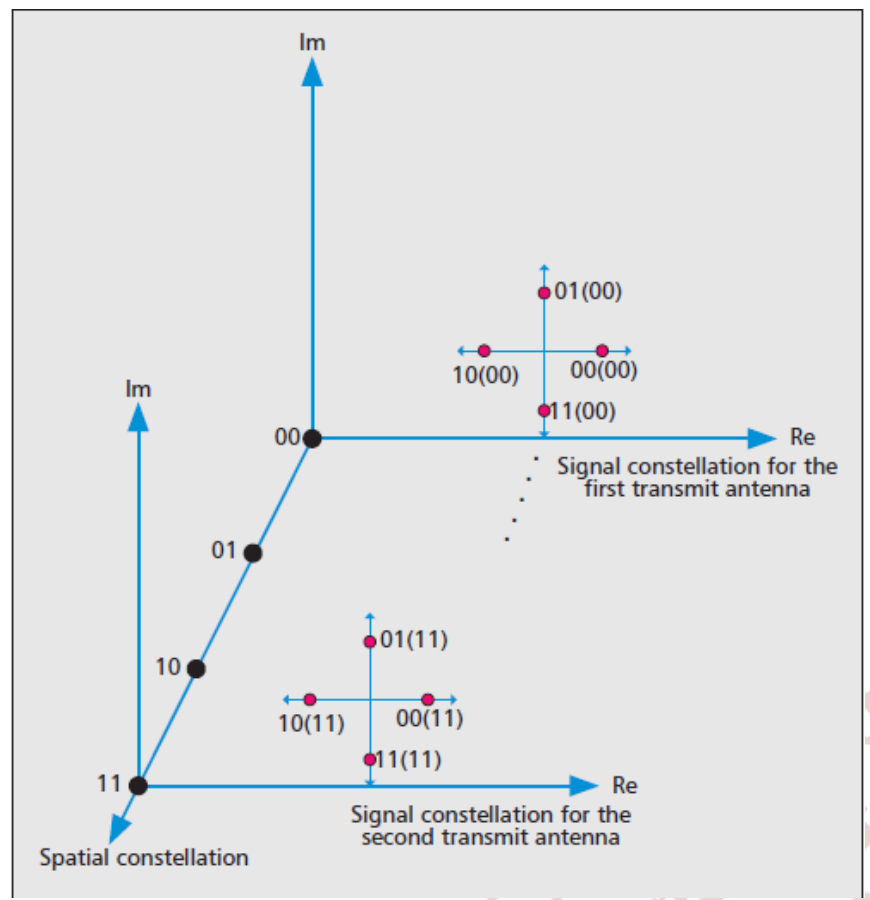

Fig. 4 Constellation Diagram

\section{Device-to-Device (D2D) communication}

D2D will enable mobile devices to discover the presence of other devices in their vicinity and to communicate with them directly, with minimal involvement from the network. Major Internet players, including Facebook, Google and Twitter, have acquired or developed location-based components for their online platforms. Current approaches generally rely on an application tracking the location of a user or device and use network resources to trigger events. LTE D2D could provide a decentralised approach to proximity discovery and device-to-device communication, which is efficient, flexible, dynamic and secure, to enable proximitybased services to flourish. Local-area device-to-device communication ex:, WiFi and Bluetooth are available. However, these technologies have shortcomings.

Unlicensed spectrum: WiFi and Bluetooth operate in unlicensed spectrum, without any centralised control of usage or interference. This is not generally a problem when usage densities are low, but it would become a major limitation as proximity-based services proliferate. Throughput, range and reliability would all suffer.

Manual pairing $\mathrm{WiFi}$ and Bluetooth rely on manual pairing of devices to enable communication between them, which would be a serious stumbling block for autonomous, dynamic proximity-based services
Security The security features of WiFi and Bluetooth are much less robust than those used in public cellular systems

Independence from cellular networks $\mathrm{WiFi}$ and Bluetooth operate independently from cellular radio technology such as LTE. Any form of device-todevice discovery based on them would have to run in parallel with cellular radio operation, which would be insufficient and would become significant drain on device batteries.

Some of the potential benefits of LTE D2D include

Radio resource management:Unlike Bluetooth and WiFi, LTE operates in licensed spectrum and the radio resources are carefully managed by the network, to minimise interference and maximise the performance of the system. The same mechanisms can be extended to D2D

Performance: Direct communication between nearby devices may be able to achieve even higher throughput and lower latency than communication through an LTE base station.

Spectrum reuse: D2D could enable even tighter reuse of spectrum than can be achieved by LTE small cells, by confining radio transmissions to the point-topoint connection between two devices

Network load : Relieving the base stations and other network components of an LTE network of some of their traffic-carrying responsibilities.

Energy efficiency: Integrating D2D into the LTE system provides the opportunity to achieve energyefficient device.

Security: D2D can take advantage of the key generation and distribution mechanisms already available in LTE, to achieve high levels of security.

Standardisation: Incorporating D2D into the LTE standard will provide a common set of tools for proximity-based services, rather than a disparate set of approaches by different application providers.

D2D is termed Proximity Services (ProSe) and has two main components, illustrated in the figure below 

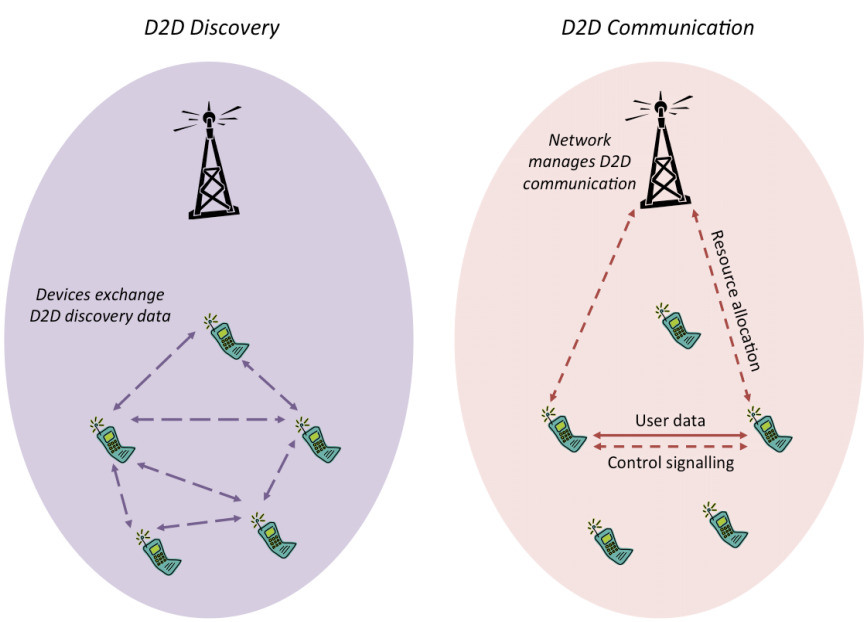

Fig 5: Device to Device Communication

D2D Discovery enables a mobile device to use the LTE radio interface to discover the presence of other D2D-capable devices in its vicinity and, where permitted, to ascertain certain information about them.

D2D Communication is the facility for D2D mobile devices to use the LTE radio interface to communicate directly with each other, without routing the traffic through the LTE network. The network exerts a light touch by controlling the radio resource allocation and security of connection.

\section{Internet of Things (IoT)}

Internet of Things (IoT) will help moving beyond standalone devices into a new era where everything is connected. The Internet of things is the network of physical devices, vehicles, home appliances and other items embedded with electronics, software, sensors, actuators, and connectivity which enable these objects to connect and exchange data. Promising technology to improve utilisation of congested spectrum.

\section{E. Cognitive Radio}

In $\mathrm{CR}$ secondary user can share spectrum with licensed primary system. Secondary user can use the spectrum when not in use CR receivers to monitor and allocate unused spectrum through spectrum sensing, and feed information back to CR Transmitter Interference tolerance basis Can share spectral resources by keeping interference below threshold Achieves enhanced spectrum and energy efficiency

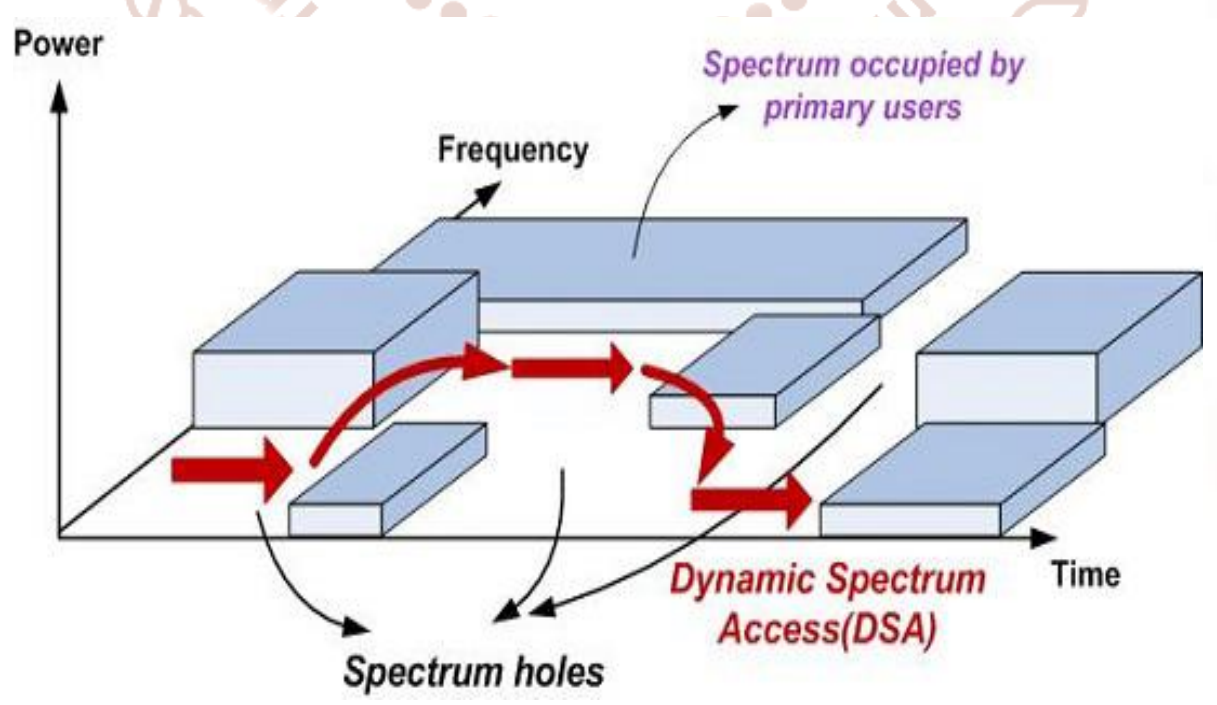

Fig 6: Dynamic Spectrum access 


\section{F. Small Cell}

Traditional capacity expansion techniques (e.g., cell splitting) are insufficient and introduce a huge CAPEX. Small cells Provides fast, flexible, and costefficient solution, Low-powered radio access nodes which can operate in licensed and/or unlicensed spectrum bands range in meters.

Small cells include femtocells, picocells, microcells, and metrocells

\section{Femtocells}

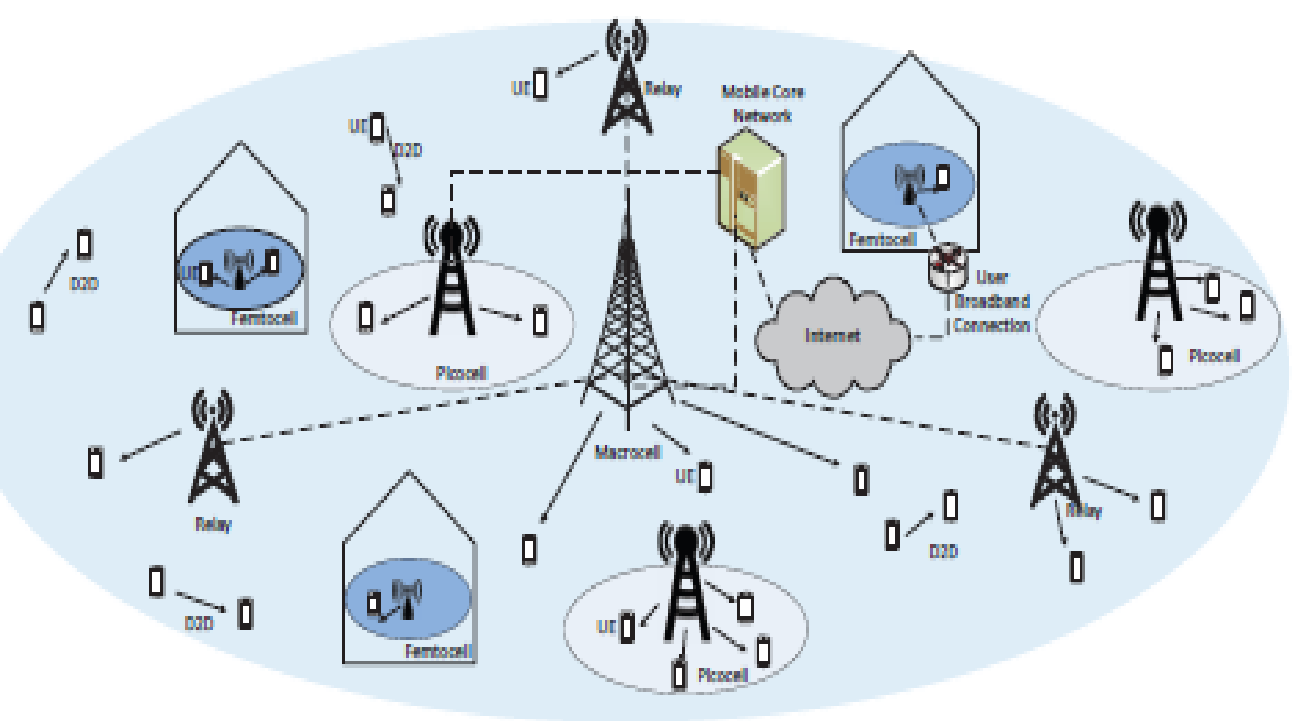

Fig 7: Cellular Wireless Network

Small area covered by a small base station femto access point (FAP),intended for residential indoor applications installed and managed by customer. Key attributes are IP backhaul, self-optimization, low power consumption, ease of deployment (userdeployed), closed/open/hybrid access

\section{Picocell:}

Low-power compact base stations, used in enterprise or public indoor areas, sometimes encompasses outdoor small cells as well. Key attributes are Wired or wireless backhaul, operator deployed, self optimization, open access.

\section{Micro Cell}

Outdoor short-range base station aiming at enhancing coverage for both indoor and outdoor users Key attributes are wired or wireless backhaul, self optimization, low power consumption, open access

\section{Metro Cell}

Small cell technologies designed for high-capacity metropolitan areas, typically installed on building walls, lampposts . Key attributes are wired or wireless backhaul, operator deployed, self optimization, open access relays . 


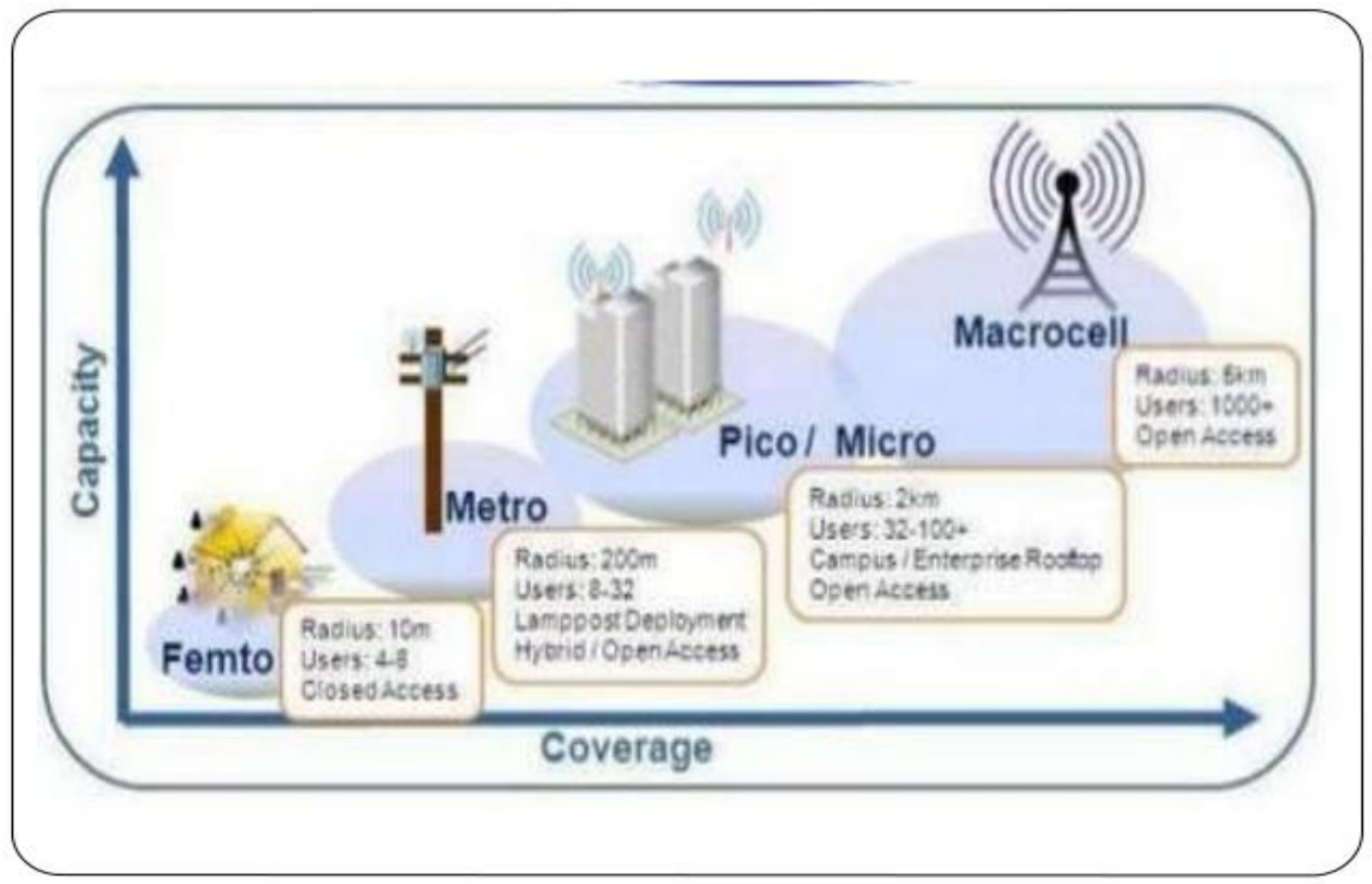

\section{Fig 7 comparison of cell}

\section{CONCLUSION}

In this paper, a detailed survey has been done on the performance requirement of $5 \mathrm{G}$ cellular communication system. Architecture has been explained with device to device communication, massive MIMO, Small cell, IoT. This paper give idea related with challenge that have in $5 \mathrm{G}$ and various emerging technology. Also provide the idea for researchers for next generation network.

\section{REFERENCES}

1. J. G. Andrews, A. Ghosh, and R. Muhamed, Fundamentals of WiMAX. Englewood Cliffs, NJ, USA: Prentice-Hall, 2007.

2. M. Peng, C. Wang, J. Li, H. Xiang, and V. Lau, "Recent advances in underlay heterogeneous networks: Interference control, resource allocation and self-organization," IEEE Commun. Surveys Tuts., vol. 17, no. 2,pp 700-729,2015

3. Y. Xu, G. Yue, and S. Mao, 'UUser grouping for massive MIMO in FDD systems: New design methods and analysis," IEEE Access, vol. 2, no. 1, pp. 947959, Sep. 2014.
4. Teguig, B. Scheers, and V. Le Nir, Wata Fusion Schemes for Cooperative Spectrum Sensing in Cognitive Radio Networks," Military commun. and information sys. Conf. (MCC), Poland, pp.17, October 2012.

5. J. Zhou, Z. Cao, X. Dong, and A. V. Vasilakos, "Security and Privacy for Cloud-Based IoT: Challenges," IEEE Communications Magazine, vol. 55, no. 1, pp. 26-33, 2017.

6. Ali, G. A. Shah, M. O. Farooq, and U. Ghani, "Technologies and challenges in developing Machine-to-Machine applications: a survey," Journal of Network and Computer Applications, vol. 83,no. 4, pp. 124-139, 2017.

7. S. K. Datta, C. Bonnet, and N. Nikaein, "An IoT gateway centric architecture to provide novel M2M services," in Internet of Things (WF-IoT), 2014 IEEE World Forum on, 2014, pp. 514-519: IEEE.

8. Y. Yan, Y. Qian, H. Sharif, and D. Tipper, "A survey on smart grid communication infrastructures: Motivations, requirements and challenges," IEEE communications surveys \& tutorials, vol. 15, no. 1, pp. 5-20, 2013

9. Gomez, J. Oller, and J. Paradells, "Overview and evaluation of bluetooth low energy: An emerging 
low-power wireless technology," Sensors, vol. 12, 11. P. Reininger, "3GPP Standards for the Internet-ofno. 9, pp. 11734-11753, 2012. Things," Smart Summit Singapore, [Online].Availability:

10. W. Group, "Wireless medium access control and physical layer specifications for low-rate wireless personal area networks," IEEE Standard, vol. 802, https://www.slideshare.net/eikoseidel/3gppstandards-for-the-internetofthings, 2016. no. 4, p. 2003, 2003.

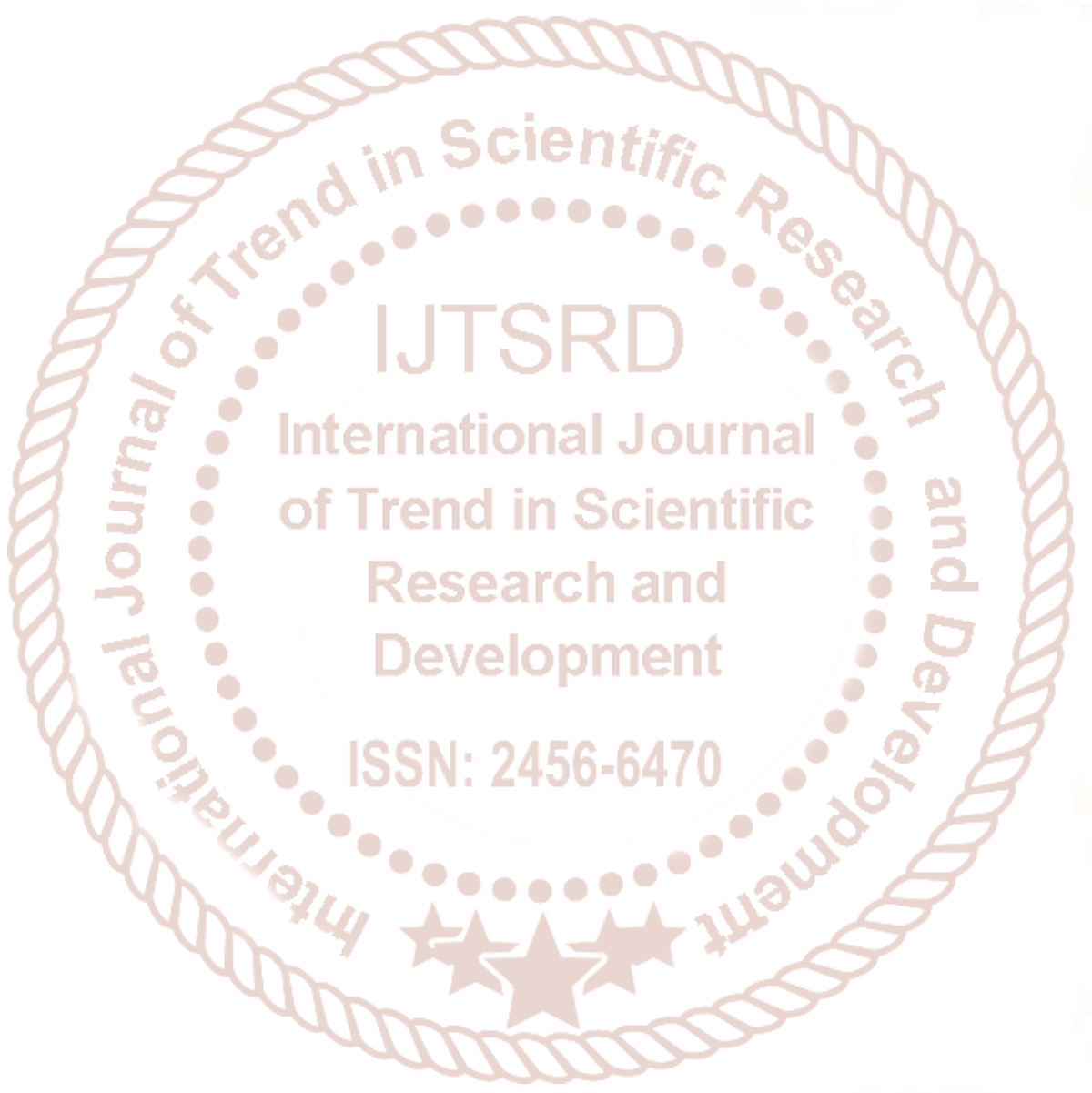

